\title{
Combined therapies with exercise, ozone and mesenchymal stem cells improve the expression of HIF1 and SOX9 in the cartilage tissue of rats with knee osteoarthritis
}

\author{
SARA ASADI ${ }^{1}$, PARVIN FARZANEGI ${ }^{1 *}$ (1) and MOHAMMAD \\ ALI AZARBAYJANI ${ }^{2}$
}

${ }^{1}$ Department of Exercise Physiology, Sari Branch, Islamic Azad University, Sari, Iran

${ }^{2}$ Department of Sport Physiology, Central Tehran Branch, Islamic Azad University, Tehran, Iran

Received: April 14, 2019 • Accepted: March 6, 2020

Published online: July 24, 2020

(C) 2020 Akadémiai Kiadó, Budapest

\begin{abstract}
Purpose: Knee osteoarthritis $(\mathrm{OA})$ is a common type of degenerative joint disease which decreases the quality of life. Sex-determining region Y box 9 (SOX9) and hypoxia-inducible factor-1 (HIF1) are considered as the key regulators of OA. We investigated the effect of combined therapies with mesenchymal stem cells (MSCs), ozone $\left(\mathrm{O}_{3}\right)$ and exercise training on SOX9 and HIF1 expression in the cartilage of rats with knee OA. Methods: Knee OA was induced by surgical method. OA rats were divided into model, MSCs, ozone, exercise, MSCs + ozone, MSCs + exercise, ozone + exercise and MSCs + ozone + exercise groups. Rats in the MSCs group received intraarticular injection of $1 \times 10^{6} \mathrm{cells} / \mathrm{kg}$. Rats in the ozone group received $\mathrm{O}_{3}$ at the concentration of $20 \mu \mathrm{g} / \mathrm{mL}$, once weekly for 3 weeks. Rats in the exercise group were trained on rodent treadmill three times per week. 48 hours after the programs, cartilage tissues were isolated and the expression of SOX9 and HIF1 was determined using Real-Time PCR. Results: Significant differences were found in the expression of SOX9 and HIF1 between groups $(P<0.0001)$. Although combined therapies with exercise, MSCs and $\mathrm{O}_{3}$ significantly increased the expression of SOX9 and HIF1 in the cartilage tissue of rats with knee OA, combination of exercise with $\mathrm{O}_{3}$ was significantly more effective compared to the other combined therapies $(P<0.001)$. Conclusions: Combined therapy with exercise, MSCs and $\mathrm{O}_{3}$ significantly increased the expression of SOX9 and HIF1 genes in the cartilage of rats with knee OA; however, exercise $+\mathrm{O}_{3}$ was significantly more effective.
\end{abstract}

" Corresponding author. Department of Exercise Physiology, Sari Branch, Islamic Azad University, Sari, Iran. Postal Code: 4816613485, Tel: +98 9112230233; E-mail: parvin.farzanegi@gmail.com 


\section{KEYWORDS}

knee osteoarthritis, $0_{3}$ therapy, MSCs therapy, exercise training, SOX9, HIF1

\section{INTRODUCTION}

Osteoarthritis (OA) is a degenerative joint disease characterized by chronic pain, stiffness, and erosion in the cartilage, which subsequently decreases the quality of life in patients. Although it affects the joints in fingers, wrists, ankles, and hips, knee OA is the most common form of articular disease which affects $13 \%$ of women and $10 \%$ of men aged 60 years [1]. Recent reports demonstrated that more than 20 million people in the United States suffer from OA [2]. Genetics, obesity or overweight, life style, repetitive movements or joints injuries, inflammatory reactions and oxidative stress, as well as several metabolic disorders such as diabetes, cancer, hemochromatosis, and acromegaly are significant risk factors for increased risk of knee OA [1, 3]. Although the incidence of knee $\mathrm{OA}$ is high, the exact cellular and molecular mechanism of the disease development and progression is not well-understood. For this reason, unfortunately, there is no definitive medication for the prevention of knee OA, and current treatments only ameliorate signs and symptoms without a significant improvement.

Recent studies have demonstrated that various genes regulate the development and progression of OA [4-6]. Dysregulation of the genes involved can be associated with severe damages in the cartilage tissue and consequently articular diseases such as knee OA. Therefore, identification of genes and their expression patterns in patients are valuable and provide a precondition for the treatment and improvement of the disease and the quality of life in these patients. Sex-determining region Y box 9 (SOX9) is a cartilage-specific transcription factor, which plays an important role in the regulation of the expression of many extracellular matrix (ECM) proteins such as collagen type II [7]. Recent studies reported that decreased expression of SOX9 can be associated with severe cartilage damage and knee OA [8]; however, the exact mechanism of its action is unclear. In addition to SOX9, hypoxia-inducible factor-1 (HIF1) is another significant transcription factor which is highly expressed in articular chondrocytes [9]. It is a heterodimeric factor consisting of HIF- $1 \beta$ and HIF- $1 \alpha$ subunits [9]. Previous studies demonstrated that mechanical stress and pro-inflammatory cytokines contribute to OA pathogenesis through the dysregulation of HIF1 [10]. These data indicate that HIF1 and SOX9 are critical factors in the development of OA and inflammatory reactions [11]. Therefore, these gene products might be targets in a possible therapy. Nevertheless, little is known about SOX9 and HIF1 expression or the effect of therapeutic methods on the expression of these genes at the early stage of OA. For this reason, in this research we evaluated SOX9 and HIF1 expression in the cartilage of rats with knee OA, followed by studying the effect of mesenchymal stem cells (MSCs), ozone $\left(\mathrm{O}_{3}\right)$ and exercise training on the expression of these genes.

Ozone therapy is a safe and effective method, which has frequently been used for the treatment of OA [12]. More recently, Feng and Beiping [13] revealed that $\mathrm{O}_{3}$ therapy importantly reduces pain intensity and improves the quality of life in patients with knee OA. In addition, several lines of studies indicated that MSCs can safely improve cartilage damages and 
knee OA due to their immunomodulatory and anti-inflammatory activities [14]. Although MSCs have strong immunosuppressive activity, their therapeutic advantage in OA primarily lies in stimulating the differentiation ability of chondrocytes, and hence building up new cartilage [15]. Interestingly, mild to moderate exercise training has been implicated for the treatment of knee OA by many researchers [16]. Clinical trials studies showed that regular and mild exercises can reduce pain severity and improve quality of life, walking disability, stair climbing, and sit-up speed in patients with knee OA [17]. Although these studies indicated the importance of exercise training, $\mathrm{O}_{3}$ and MSCs therapies in the improvement of $\mathrm{OA}$ and quality of life, the exact mechanism of these methods is not clear. Given the critical role of SOX9 and HIF1 in OA, we assume that MSCs and $\mathrm{O}_{3}$ therapy along with exercise training may be effective through the improvement of SOX9 and HIF1 expression. Therefore, we designed this study to compare the effect of MSCs and $\mathrm{O}_{3}$ therapy along with exercise training on SOX9 and HIF1 expression and histological outcomes in rats with knee OA.

\section{MATERIALS AND METHODS}

\section{Experimental animals}

In this experimental study, 63 male Wistar rats (between 40 and 45 weeks of age with body weights of 250-300 g) were provided by the Laboratory Animal Research Center at the Islamic Azad University of Sari. Rats were housed in standard shoebox cages $(42 \times 26.5 \times 15 \mathrm{~cm}), 3$ per cage in a climate-controlled room (ambient temperature of $22 \pm 2{ }^{\circ} \mathrm{C}$, humidity $50 \pm 5 \%$, and a 12:12-h light/dark cycle). Rats were fed with standard diet and water. The study was approved by the Animal Care and Use Committee at Islamic Azad University, Sari branch.

\section{Induction of knee $O A$ in experimental rats}

OA was induced through the surgical method according to a previously published report [18]. Before OA induction, rats were anesthetized with ketamine $(30-50 \mathrm{mg} / \mathrm{kg}$ ) and xylasine (3-5 $\mathrm{mg} / \mathrm{kg}$ ). To expose the knee joint, a 1-cm longitudinal incision was made and then the knee joint was shortly opened through lateral dislocation of the patella and the patellar ligament. A longitudinal cut was provided in the knee joint capsule through the medial parapatellar incision. An incomplete incision was made through the medial meniscotibial ligament without articular cartilage and other ligament injury. Finally, the knee joint capsule and then the skin were closed with a 6-0 absorbable suture and 6-0 silk suture, respectively.

Before starting the MSCs, exercise and $\mathrm{O}_{3}$ therapies, these rats were fed with standard food and water for three weeks. Rats with knee OA were then randomly divided into 8 groups ( 7 rats in each group), including: patient or model (OA rats without any treatments), MSCs therapy, ozone therapy, exercise training, MSCs $+\mathrm{O}_{3}$ therapy, MSCs + exercise therapy, exercise $+\mathrm{O}_{3}$ therapy, and MSCs $+\mathrm{O}_{3}+$ exercise groups. Rats in combined therapies were first treated with MSCs, then with $\mathrm{O}_{3}$, and finally with exercise training. An additional group known as control group was also entered into the study. Rats in the control group were healthy and did not receive any treatments. 


\section{MSCs injection}

Bone marrow-derived MSCs were purchased from the Histogenotech Company (Tehran, Iran). MSCs were extracted from healthy male Wistar rats (25-300 g). These MSCs were purchased when they reached $>90 \%$ confluency at passages 3 or 4 . MSCs were intraarticularly injected to the right knee at a concentration of $1 \times 10^{6}$ cells $/ \mathrm{kg}$. Rats in the MSCs group received only a single dose of injection.

\section{Ozone therapy}

Ozone was produced from medical-grade oxygen $\left(\mathrm{O}_{2}\right)$ by OZOMED 01 equipment. It was generated through a silent electric discharge, and its concentration was measured using a UV spectrophotometer at $254 \mathrm{~nm}$. Ozone was injected into the knee through the tibiofemoral joint line at a concentration of $20 \mu \mathrm{g} / \mathrm{mL}$, once weekly for 3 weeks, starting 3 weeks after the modeling.

\section{Exercise program}

Before exercise training, rats in this group were adapted to treadmill running for 5 days (once a week, with $\mathrm{VO}_{2} \max 60-70 \%$, speed of $16 \mathrm{~m} / \mathrm{min}$ at $0 \%$ inclination for $10 \mathrm{~min} /$ day). The exercise program was started with a 30 -min run on the treadmill without slope and a speed of $16 \mathrm{~m} / \mathrm{min}$ in the first week, which was progressively increased to 30 minutes by the third week. Warm-up and cool-down times were done for $5 \mathrm{~m} / \mathrm{min}$ at the beginning and the end of the exercise period.

\section{Samples collection and gene expression analysis}

Forty-eight hours after the end of programs, all rats were anesthetized with ketamine (30-50 $\mathrm{mg} / \mathrm{kg}$ ) and xylasine (3-5 mg/kg). Cartilage tissues were isolated and a piece of tissue was used for histological examination. Tissues were fixed in $10 \%$ formalin for $48 \mathrm{~h}$ and fragments were then dehydrated in a graded series of ethanol, embedded in paraffin and sectioned using an automatic microtome (ROTOCUT200, SCILAB Company, England) at $5 \mathrm{~mm}$ thickness. The sectioned tissues were stained with haematoxylin-eosin ( $\mathrm{H} \& \mathrm{E})$ and their morphological and histological parameters were examined by light microscopy.

lFor gene expression analysis, a fragment of cartilage tissue $(\sim 50 \mathrm{mg})$ was homogenized in phosphate buffered saline (0.01 M; pH 7.0) at $4{ }^{\circ} \mathrm{C}$ with a homogenizer (Hielscher, UP100H). Total RNAs were then extracted using the RNX-Plus (SinaClon; RN7713C) Kit. The quantity and quality of the extracted RNAs were characterized using a Nanodrop ND-1000 spectrophotometer (Thermo Sci., Newington, NH) method. cDNA was synthesized from RNA samples using Revert Aid Reverse Transcriptase (Thermo science, Germany) at $42{ }^{\circ} \mathrm{C}$ for $1 \mathrm{~h}$ with random hexamer primers (Thermo science, Germany). A Rotor Gene 6,000 (Corbett Research, Australia) thermocycler was applied for amplifications. Each reaction included $5 \mu$ master mix and $100 \mathrm{~nm}$ primers. Primer sequences were synthesized as follows: SOX9, 5'-AGGAAGTCGGTGAAGAATGGG-3' (forward), $\quad 5^{\prime}$-GGTTTTGGGAGTGGTGGGT-3' (reverse); HIF1, 5'-GTTGTTGTTGTTGTCTGTGGG-3' (forward), 5'-AGTGAAAATGAAGGAGGAAGGG-3' (reverse), and glyceraldehyde 3-phosphate dehydrogenase (GAPDH), $5^{\prime}$ AAGTTCAACGGCACAGTCAAGG-3' (forward); 5'-CATACTCAGCACCAGCATCACC-3' (reverse), as a reference gene. The mRNA levels of HIF1 and SOX9 were normalized relative to the amount of $G A P D H$ mRNA. Delta $C t(\triangle \mathrm{CT})$ was calculated using the following formula 
according to the method described by Livak and Schmittgen [19]: [ $\Delta \mathrm{CT}=\mathrm{CT}$ (target) $-\mathrm{CT}$ ]. The expression profile of each gene was determined in triplicates. We determined the mean of $\mathrm{Ct}$ of each gene and then calculated delta-Ct and, on that basis, fold change by calculating $2^{-\Delta \mathrm{Ct}}$ [19].

\section{Statistical analysis}

The mean expression of HIF1 and SOX9 between all groups was compared using the One-Way ANOVA: Post Hoc-Tukey test. SPSS software (IBM, version 19, USA) was applied for analysis of data. A $P<0.05$ was considered as significant.

\section{RESULTS}

Chondroblasts and chondrocytes were arranged regularly inside the cavities in the normal group (Fig. 1). The space between each cartilage cell and ECM showed as a colored aura around the cell, suggesting the existence of glycoproteins around the chondrocyte cavities. Bone tissue was
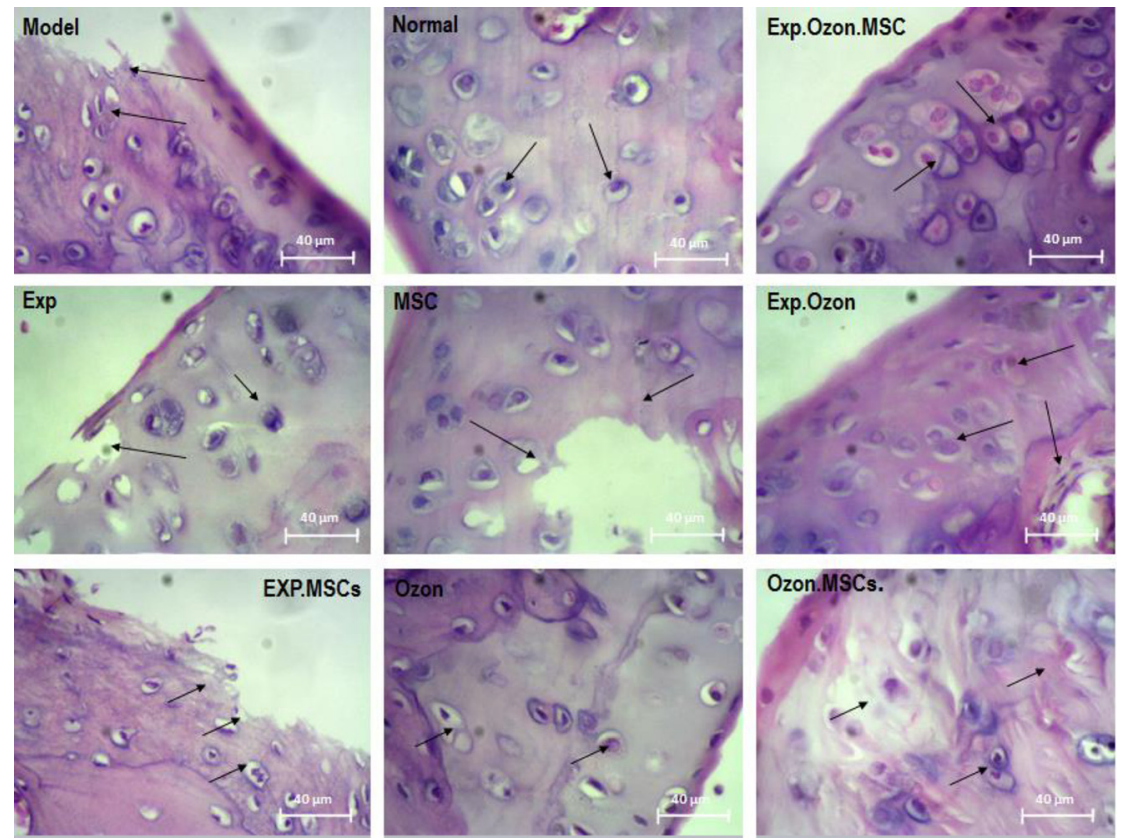

Fig. 1. Histological examination of cartilage in each study group by H\&E staining. Chondroblasts and chondrocytes were arranged regularly inside the cavities in the normal group. In the model group, chondrocyte cells were destroyed. Moderate abnormalities are observed in the $\mathrm{O}_{3}$, MSCs exercise training groups. The proliferating chondrocyte cells were observed in combined therapies with MSC, ozone and exercise, and injured cartilaginous tissues were completely replaced with normal tissues. EXP: exercise training 
normal in structure and had cavities consisting of bone marrow tissue and regular arrangement of osteocyte cells. Synovial membranes, lateral ligaments and retaining muscles around the joint and articular capsules had normal structure (Fig. 1).

In the model group, chondrocyte cells were destroyed, which was observed in the synovial membrane, lateral ligaments and retaining muscles. Articular joint and collateral ligament injuries with apoptotic chondrocytes were also observed. A small number of isogenous groups were found, which indicates mild proliferation of cartilage cells in this group (Fig. 1). Although treatment with $\mathrm{O}_{3}$, MSCs exercise training alone slightly improved these abnormalities in the cartilage and bone tissues, moderate abnormalities can be seen in these groups (Fig. 1). Combined therapies with MSC, ozone and exercise improved these abnormalities more than in the other groups. Proliferating chondrocyte cells were observed within the lacunae as isogenic groups, in even higher numbers than in the healthy group. Injured cartilaginous tissues were completely replaced with normal tissues. The articular capsule of the knee joint and the medial ligament were well-repaired (Fig. 1).

Comparison of HIF1 expression in all groups is shown in Fig. 2. The ANOVA test analysis showed significant differences in the expression of $H I F 1$ between groups $(P<$ 0.0001). The control group had significantly higher expression of HIF1 compared to the model (2,529.42-fold; $P<0.001)$, MSCs (17.32-fold; $P<0.001)$, exercise (20.02-fold; $P<$ 0.001 ), ozone (75.19-fold; $P<0.001)$, MSCs $+\mathrm{O}_{3}$ (1.85-fold; $\left.P<0.001\right)$, MSCs + exercise (6.28-fold; $P<0.001)$, MSCs $+\mathrm{O}_{3}+$ exercise $(1.92$-fold; $P<0.001)$ groups (Table 1$)$. Although exercise training, MSCs and $\mathrm{O}_{3}$ therapies increased the expression of HIF1 in the cartilage tissue of OA rats, this improvement was not statically significant (Fig. 2). Although combined therapies with exercise, MSCs and $\mathrm{O}_{3}$ significantly increased the expression of $H I F 1$, combination of exercise with $\mathrm{O}_{3}$ was significantly more effective compared to the other combined therapies (Fig. 2).

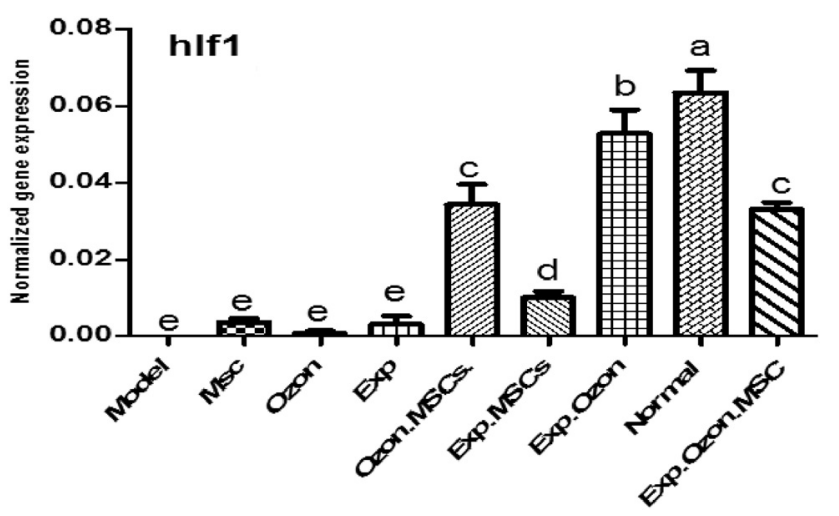

Fig. 2. Comparison of the mean mRNA levels of HIF. Gene expression was detected by Real-Time PCR. There was no significant difference in the mRNA levels of HIF between groups with similar MSC: mesenchymal stem cell; Exp: exercise training symbols (a-e). The order of the mean mRNA levels of HIF was $\mathrm{a}>\mathrm{b}>\mathrm{c}>\mathrm{d}>\mathrm{e}$. One-Way ANOVA: Post Hoc-Tukey test was applied to compare the mean values of HIF expression pattern between all groups. 
Table 1. Fold change ratio of HIF1 expression in each group

\begin{tabular}{|c|c|c|c|}
\hline & Fold-change ratio & Up-/down-regulation & $P$-value \\
\hline Control vs. Model & $2,529.42$ & Up-regulated & $<0.001$ \\
\hline MSCs vs. Model & 14.67 & Up-regulated & 0.46 \\
\hline $\mathrm{O}_{3}$ vs. Model & 3.64 & Up-regulated & 0.86 \\
\hline Exp vs. Model & 12.63 & Up-regulated & 0.52 \\
\hline MSCs $+\mathrm{O}_{3}$ vs. Model & $1,369.48$ & Up-regulated & $<0.001$ \\
\hline MSCs + Exp vs. Model & 403.06 & Up-regulated & 0.048 \\
\hline Exp $+\mathrm{O}_{3}$ vs. Model & $2,102.61$ & Up-regulated & $<0.001$ \\
\hline MSCs $+\mathrm{O}_{3}+$ Exp vs. Model & $1,319.89$ & Up-regulated & $<0.001$ \\
\hline Control vs. MSCs & 17.32 & Up-regulated & $<0.001$ \\
\hline Control vs. $\mathrm{O}_{3}$ & 75.19 & Up-regulated & $<0.001$ \\
\hline Control vs. Exp & 20.02 & Up-regulated & $<0.001$ \\
\hline Control vs. MSCs $+\mathrm{O}_{3}$ & 1.85 & Up-regulated & $<0.001$ \\
\hline Control vs. MSCs + Exp & 6.28 & Up-regulated & $<0.001$ \\
\hline Control vs. $\operatorname{Exp}+\mathrm{O}_{3}$ & 1.20 & Up-regulated & 0.036 \\
\hline Control vs. MSCs $+\mathrm{O}_{3}+\operatorname{Exp}$ & 1.92 & Up-regulated & 0.000 \\
\hline MSCs vs. $\mathrm{O}_{3}$ & 4.34 & Up-regulated & 0.57 \\
\hline MSCs vs. Exp & 1.16 & Up-regulated & 0.92 \\
\hline Exp vs. $\mathrm{O}_{3}$ & 3.76 & Up-regulated & 0.63 \\
\hline $\mathrm{O}_{3}+$ Exp vs. MSCs & 14.39 & Up-regulated & $<0.001$ \\
\hline $\mathrm{O}_{3}+$ Exp vs. Exp & 16.64 & Up-regulated & $<0.001$ \\
\hline $\mathrm{O}_{3}+\operatorname{Exp}$ vs. $\mathrm{O}_{3}$ & 62.50 & Up-regulated & $<0.001$ \\
\hline $\mathrm{O}_{3}+$ Exp vs. MSCs $+\mathrm{O}_{3}$ & 1.54 & Up-regulated & $<0.001$ \\
\hline $\mathrm{O}_{3}+$ Exp vs. MSCs $+\operatorname{Exp}$ & 5.22 & Up-regulated & $<0.001$ \\
\hline $\mathrm{O}_{3}+\operatorname{Exp}$ vs. MSCs $+\mathrm{O}_{3}+\operatorname{Exp}$ & 1.59 & Up-regulated & $<0.001$ \\
\hline MSCs $+\mathrm{O}_{3}$ vs. MSCs & 9.38 & Up-regulated & $<0.001$ \\
\hline MSCs $+\mathrm{O}_{3}$ vs. Exp & 10.84 & Up-regulated & $<0.001$ \\
\hline MSCs $+\mathrm{O}_{3}$ vs. $\mathrm{O}_{3}$ & 40.71 & Up-regulated & $<0.001$ \\
\hline MSCs $+\mathrm{O}_{3}$ vs. MSCs + Exp & 3.40 & Up-regulated & $<0.001$ \\
\hline MSCs $+\mathrm{O}_{3}$ vs. MSCs $+\mathrm{O}_{3}+\operatorname{Exp}$ & 1.04 & Up-regulated & 0.8 \\
\hline MSCs + Exp vs. Exp & 3.19 & Up-regulated & 0.16 \\
\hline MSCs + Exp vs. $\mathrm{O}_{3}$ & 11.98 & Up-regulated & 0.068 \\
\hline MSCs + Exp vs. MSCs & 2.76 & Up-regulated & 0.19 \\
\hline MSCs + Exp vs. MSCs $+\mathrm{O}_{3}+\operatorname{Exp}$ & 3.27 & Down-regulated & $<0.001$ \\
\hline MSCs $+\mathrm{O}_{3}+$ Exp vs. $\mathrm{O}_{3}$ & 39.24 & Up-regulated & $<0.001$ \\
\hline MSCs $+\mathrm{O}_{3}+$ Exp vs. Exp & 10.44 & Up-regulated & $<0.001$ \\
\hline MSCs $+\mathrm{O}_{3}+$ Exp vs. MSCs & 9.04 & Up-regulated & $<0.001$ \\
\hline
\end{tabular}

${ }^{*} P<0.05$ is considered as significant; One-Way ANOVA: Post Hoc-Tukey test was applied to compare the mean value of HIF1 expression pattern between all groups.

Comparison of $S O X 9$ expression is shown in Fig. 3. Significant differences were found in the expression of SOX9 between groups $(P<0.0001)$. The control group had significantly higher expression of SOX9 compared to the model (52.65-fold; $P=0.000)$, MSCs (7.14-fold; $P=$ $0.001)$, ozone (3.37-fold; $P=0.000)$, exercise (11.51-fold; $P=0.000)$, MSCs + exercise (8.81fold; $P=0.000)$, MSCs $+\mathrm{O}_{3}(1.83$-fold; $P=0.000)$, and MSCs $+\mathrm{O}_{3}+$ exercise (1.4-fold; $P=$ 
Table 2. Fold change ratio of $S O X 9$ expression in each group

\begin{tabular}{|c|c|c|c|}
\hline & Fold-change ratio & Up-/down-regulation & $P$-value \\
\hline Control vs. Model & 52.65 & Up-regulated & $<0.001$ \\
\hline MSCs vs. Model & 7.37 & Up-regulated & 0.15 \\
\hline $\mathrm{O}_{3}$ vs. Model & 15.62 & Up-regulated & 0.002 \\
\hline Exp vs. Model & 4.58 & Up-regulated & 0.42 \\
\hline MSCs $+\mathrm{O}_{3}$ vs. Model & 28.77 & Up-regulated & $<0.001$ \\
\hline MSCs + Exp vs. Model & 5.98 & Up-regulated & 0.26 \\
\hline $\mathrm{Exp}+\mathrm{O}_{3}$ vs. Model & 45.63 & Up-regulated & $<0.001$ \\
\hline MSCs $+\mathrm{O}_{3}+$ Exp vs. Model & 37.64 & Up-regulated & $<0.001$ \\
\hline Control vs. MSCs & 7.14 & Up-regulated & $<0.001$ \\
\hline Control vs. $\mathrm{O}_{3}$ & 3.37 & Up-regulated & $<0.001$ \\
\hline Control vs. Exp & 11.51 & Up-regulated & $<0.001$ \\
\hline Control vs. MSCs $+\mathrm{O}_{3}$ & 1.83 & Up-regulated & $<0.001$ \\
\hline Control vs. MSCs + Exp & 8.81 & Up-regulated & $<0.001$ \\
\hline Control vs. $\operatorname{Exp}+\mathrm{O}_{3}$ & 1.15 & Up-regulated & 0.12 \\
\hline Control vs. MSCs $+\mathrm{O}_{3}+\operatorname{Exp}$ & 1.40 & Up-regulated & 0.002 \\
\hline MSCs vs. $\mathrm{O}_{3}$ & 2.12 & Down-regulated & 0.07 \\
\hline MSCs vs. Exp & 1.61 & Up-regulated & 0.53 \\
\hline Exp vs. $\mathrm{O}_{3}$ & 3.41 & Down-regulated & 0.017 \\
\hline $\mathrm{O}_{3}+$ Exp vs. MSCs & 6.19 & Up-regulated & $<0.001$ \\
\hline $\mathrm{O}_{3}+$ Exp vs. Exp & 9.97 & Up-regulated & $<0.001$ \\
\hline $\mathrm{O}_{3}+$ Exp vs. $\mathrm{O}_{3}$ & 2.92 & Up-regulated & $<0.001$ \\
\hline $\mathrm{O}_{3}+$ Exp vs. MSCs $+\mathrm{O}_{3}$ & 1.59 & Up-regulated & 0.001 \\
\hline $\mathrm{O}_{3}+$ Exp vs. MSCs + Exp & 7.64 & Up-regulated & $<0.001$ \\
\hline $\mathrm{O}_{3}+$ Exp vs. MSCs $+\mathrm{O}_{3}+\operatorname{Exp}$ & 1.21 & Up-regulated & 0.079 \\
\hline MSCs $+\mathrm{O}_{3}$ vs. MSCs & 3.90 & Up-regulated & $<0.001$ \\
\hline MSCs $+\mathrm{O}_{3}$ vs. Exp & 6.29 & Up-regulated & $<0.001$ \\
\hline MSCs $+\mathrm{O}_{3}$ vs. $\mathrm{O}_{3}$ & 1.84 & Up-regulated & 0.005 \\
\hline MSCs $+\mathrm{O}_{3}$ vs. MSCs + Exp & 4.82 & Up-regulated & $<0.001$ \\
\hline MSCs $+\mathrm{O}_{3}$ vs. MSCs $+\mathrm{O}_{3}+\operatorname{Exp}$ & 1.31 & Down-regulated & 0.053 \\
\hline MSCs + Exp vs. Exp & 1.31 & Up-regulated & 0.75 \\
\hline MSCs + Exp vs. $\mathrm{O}_{3}$ & 2.61 & Down-regulated & 0.036 \\
\hline MSCs + Exp vs. MSCs & 1.23 & Down-regulated & 0.75 \\
\hline MSCs + Exp vs. MSCs $+\mathrm{O}_{3}+\operatorname{Exp}$ & 6.30 & Down-regulated & $<0.001$ \\
\hline MSCs $+\mathrm{O}_{3}+$ Exp vs. $\mathrm{O}_{3}$ & 2.41 & Up-regulated & $<0.001$ \\
\hline MSCs $+\mathrm{O}_{3}+$ Exp vs. Exp & 8.22 & Up-regulated & $<0.001$ \\
\hline MSCs $+\mathrm{O}_{3}+$ Exp vs. MSCs & 5.10 & Up-regulated & $<0.001$ \\
\hline
\end{tabular}

${ }^{*} P<0.05$ is considered as significant; One-Way ANOVA: Post Hoc-Tukey test was applied to compare the mean value of SOX9 expression pattern between all groups.

0.002) groups (Table 2). There was no significant difference in SOX9 expression between the control and exercise $+\mathrm{O}_{3}$ groups $(P=0.12)$. Although exercise, MSCs and ozone therapies increased the expression of SOX9 in the cartilage tissue of OA rats, their combined therapy, especially exercise $+\mathrm{O}_{3}$, was significantly more effective (Fig. 3). 




Fig. 3. Comparison of the mean mRNA levels of SOX9. Gene expression was detected by Real-Time PCR. There was no significant difference in the mRNA levels of SOX9 between groups with similar symbols (ad). The order of the mean mRNA levels of SOX9 was a $>$ b $>c>d$. One-Way ANOVA: Post Hoc-Tukey test was applied to compare the mean values of SOX9 expression pattern between all groups. MSC: mesenchymal stem cell; Exp: exercise training

\section{DISCUSSION}

Here, we compared the effect of exercise training, ozone and MSCs therapies and their combination on the expression of SOX9 and HIF1 in the cartilage tissue of rats with knee OA. On histological examination, arthritic rats showed chondrocyte cells destruction possibly due to apoptosis, as well as articular joint and collateral ligament injuries. Our findings also show that knee $\mathrm{OA}$ is associated with a significant decrease in the expression of SOX9 and HIF1 in the cartilage tissue of arthritic rats. This is in agreement with previous research that showed downregulation of SOX9 and HIF1 mRNA and proteins in osteoarthritic subjects [20-22]. HIF has an important function in the control of energy production, matrix synthesis and cell survival by articular and growth-plate chondrocytes [23]. It is also crucial for chondrogenesis and regulates the maintenance of articular cartilage via the induction of anabolic factors and the repression of catabolic factors [24]. SOX9 is the other important factor which is critical for cartilage ECM formation and maintenance of the chondrocyte phenotype. Many studies reported decreased level and expression of SOX9 in OA subjects $[25,26]$. Therefore, these data suggest that downregulation of SOX9 and HIF may be a main reason for the loss of cartilage ECM and increased risk of OA. Furthermore, these factors are necessary for the maintenance of articular cartilage, and the dysregulation of these genes can cause cartilage degeneration and increased risk of OA development and progression.

According to these findings and the pathogenesis of OA, SOX9 and HIF can be considered as targets for treatment of the disease. To study the pathogenesis or therapeutic options for knee OA, we compared the effect of exercise training, MSCs and ozone therapies on SOX9 and HIF expression in the cartilage tissue of rats with knee OA. We found that combined therapies with exercise training, MSCs and $\mathrm{O}_{3}$ significantly increased the expression of SOX9 and HIF in the cartilage of osteoarthritic rats; however, combination of exercise with $\mathrm{O}_{3}$ was more effective. Furthermore, this combined therapy was associated with an increased number of proliferating 
chondrocyte cells within the lacunae and also with repair of injured cartilaginous tissues. Therefore, these data indicate the importance of these combined therapies in the treatment of knee OA disease, which is probably mediated through the increase in SOX9 and HIF expression. In support of this theory, Lindholm et al. [27] showed that exercise training increases HIF1 expression in skeletal muscle. Similarly, Mason and Johnson [28] demonstrated that endurance training causes overexpression of HIF1 in the skeletal muscle of mice.

To our knowledge, the effects of MSCs and ozone therapies on the expression of HIF1 and SOX9 in the cartilage of OA subjects have not been investigated yet. Here, we showed for the first time the effectiveness of MSCs and $\mathrm{O}_{3}$ to enhance the expression of these genes. However, several studies have reported the importance of exercise training, MSCs and ozone therapies in the treatment of knee OA. For instance, Helmark et al. [29] demonstrated that exercise training significantly increases the expression of anti-inflammatory cytokines in the articular cartilage of patients with knee OA. de Jesus et al. [30] reported that $\mathrm{O}_{3}$ therapy is effective for pain relief and improvement of quality of life in patients with knee OA [30]. A more recent study has demonstrated that $\mathrm{O}_{3}$ therapy is significantly associated with an improvement in hind-paw diameter, arthritis severity and inflammation in Wistar rats [31]. Some studies have indicated that $\mathrm{O}_{3}$ therapy may improve OA through the improvement of the antioxidant defense system and the prevention of inflammatory cytokines such as IL-6, TNF- $\alpha$ and IL-8 $[32,33]$. Our results reveal that increased expression of HIF1 and SOX9 may be another significant mechanism by which $\mathrm{O}_{3}$ therapy causes OA improvement.

Based on this study and accomplished studies, we hypothesized that combined therapy with $\mathrm{O}_{3}$, MSCs and exercise training, especially $\mathrm{O}_{3}+$ exercise, is effective to mitigate clinical and histopathological outcomes of arthritis by increasing the expression of the HIF1 and SOX9 genes in the cartilage tissue of rats with knee OA. However, histochemical analysis is essential to study the expression of these genes at the protein level and also to determine its relevance on quality of life in human subjects.

In conclusion, the findings of the current study revealed that knee OA is strongly associated with decreased expression of HIF1 and SOX9 in the cartilage tissues. Combined therapies with $\mathrm{O}_{3}$, MSCs, and exercise training, especially $\mathrm{O}_{3}+$ exercise training, are effective for the improvement of knee OA. However, we recommend another study at the protein level, as we only examined these factors at the mRNA level.

Funding: This study was supported by a grant received from the Islamic Azad University, Sari Branch, Iran.

\section{ACKNOWLEDGMENT}

This work was supported by the Exercise Physiology, Islamic Azad University, Sari Branch, Iran. We would also like to thank the staffs of the Exercise Physiology Centers of the Islamic Azad University, Sari, Iran.

\section{REFERENCES}

1. Heidari B. Knee osteoarthritis prevalence, risk factors, pathogenesis and features: part I. Caspian J Intern Med 2011; 2: 205-12. 
2. Loeser RF. The Role of Aging in the Development of Osteoarthritis. Trans Am Clin Climatol Assoc 2017; 128 : 44-54.

3. Lee KM, Chung CY, Sung KH, Lee SY, Won SH, Kim TG, et al. Risk factors for osteoarthritis and contributing factors to current arthritic pain in South Korean older adults. Yonsei Med J 2015; 56: 124-31.

4. Kamekura S, Hoshi K, Shimoaka T, Chung U, Chikuda H, Yamada T, et al. Osteoarthritis development in novel experimental mouse models induced by knee joint instability. Osteoarthritis Cartilage 2005; 13: 632-41.

5. Shen J, Li J, Wang B, Jin H, Wang M, Zhang Y, et al. Deletion of the transforming growth factor $\beta$ receptor type II gene in articular chondrocytes leads to a progressive osteoarthritis-like phenotype in mice. Arthritis Rheum 2013; 65: 3107-19.

6. Valdes AM, Spector TD, Tamm A, Kisand K, Doherty SA, Dennison EM, et al. Genetic variation in the SMAD3 gene is associated with hip and knee osteoarthritis. Arthritis Rheum 2010; 62: 2347-52.

7. Lefebvre V, de Crombrugghe B. Toward understanding SOX9 function in chondrocyte differentiation. Matrix Biol 1998; 16: 529-40.

8. Kim KI, Park YS, Im GI. Changes in the epigenetic status of the SOX-9 promoter in human osteoarthritic cartilage. J Bone Miner Res 2013; 28: 1050-60.

9. Pfander D, Cramer T, Swoboda B. Hypoxia and HIF-1 $\alpha$ in osteoarthritis. Int Orthop 2005; 29: 6-9.

10. Chang H, Shyu K, Wang B, Kuan P. Regulation of hypoxia-inducible factor-1alpha by cyclical mechanical stretch in rat vascular smooth muscle cells. Clin Sci (Lond) 2003; 105: 447-56.

11. Jung YJ, Isaacs JS, Lee S, Trepel J, Neckers L. IL-1beta-mediated up-regulation of HIF-1alpha via an NFkappaB/COX-2 pathway identifies HIF-1 as a critical link between inflam-mation and oncogenesis. FASEB J 2003; 17: 2115-7.

12. Manoto SL, Maepa MJ, Motaung SK. Medical ozone therapy as a potential treatment modality for regeneration of damaged articular cartilage in osteoarthritis. Saudi J Biol Sci 2018; 24: 672-9.

13. Feng $\mathrm{X}$, Beiping L. Therapeutic efficacy of ozone injection into the knee for the osteoarthritis patient along with oral celecoxib and glucosamine. J Clin Diagn Res 2017; 11: UC01-3.

14. Davatchi F, Sadeghi Abdollahi B, Mohyeddin M, Nikbin B. Mesenchymal stem cell therapy for knee osteoarthritis: 5 years follow-up of three patients. Int J Rheum Dis 2016; 19: 219-25.

15. Zhang R, Ma J, Han J, Zhang W, Ma J. Mesenchymal stem cell related therapies for cartilage lesions and osteoarthritis. Am J Transl Res 2019; 11: 6275-89.

16. Uusi-Rasi K, Patil R, Karinkanta S, Tokola K, Kannus P, Sievänen H. Exercise training in treatment and rehabilitation of Hip osteoarthritis: a 12-week pilot trial. J Osteoporos 2017; 2017: 3905492.

17. Nejati P, Farzinmehr A, Moradi-Lakeh M. The effect of exercise therapy on knee osteoarthritis: a randomized clinical trial. Med J Islam Repub Iran 2015; 29: 186.

18. Zhao Y, Liu B, Liu C. Establishment of a Surgically-induced Model in Mice to Investigate the Protective Role of Progranulin in Osteoarthritis. J Vis Exp 2014; 2014: e50924.

19. Livak KJ, Schmittgen TD. Analysis of relative gene expression data using real-time quantitative PCR and the 2(-Delta Delta C(T)) Method. Methods 2001; 25: 402-8.

20. Peffers MJ, Milner PI, Tew SR, Clegg PD. Regulation of SOX9 in normal and osteoarthritic equine articular chondrocytes by hyperosmotic loading. Osteoarthritis Cartilage 2010; 18: 1502-8.

21. Qing L, Lei P, Liu H, Xie J, Wang L, Wen T, et al. Expression of hypoxia-inducible factor- $1 \alpha$ in synovial fluid and articular cartilage is associated with disease severity in knee osteoarthritis. Exp Ther Med 2017; 13: 63-8.

22. Zhang Q, Ji Q, Wang X, Kang L, Fu Y, Yin Y, et al. SOX9 is a regulator of ADAMTSs-induced cartilage degeneration at the early stage of human osteoarthritis. Osteoarthritis Cartilage 2015; 23: 2259-68. 
23. Ren BF, Deng LF, Wang J, Zhu YP, Wei L, Zhou Q. Hypoxia regulation of facilitated glucose transporter-1 and glucose transporter-3 in mouse chondrocytes mediated by HIF-1alpha. Joint Bone Spine 2008; 75 : $176-81$.

24. Sakamoto J, Origuchi T, Okita M, Nakano J, Kato K, Yoshimura T, et al. Immobilization-induced cartilage degeneration mediated through expression of hypoxia-inducible factor-1alpha, vascular endothelial growth factor, and chondromodulin-I. Connect Tissue Res 2009; 50: 37-45.

25. Tew SR, Clegg PD, Brew CJ, Redmond CM, Hardingham TE. SOX9 transduction of a human chondrocytic cell line identifies novel genes regulated in primary human chondrocytes and in osteoarthritis. Arthritis Res Ther 2007; 9: R107.

26. Tew SR, Li Y, Pothacharoen P, Tweats LM, Hawkins RE, Hardingham TE. Retroviral transduction with SOX9 enhances re-expression of the chondrocyte phenotype in passaged osteoarthritic human articular chondrocytes. Osteoarthritis Cartilage 2005; 13: 80-9.

27. Lindholm ME, Rundqvist H. Skeletal muscle hypoxia-inducible factor-1 and exercise. Exp Physiol 2016; 101: 28-32.

28. Mason S, Johnson RS. The role of HIF-1 in hypoxic response in the skeletal muscle. Adv Exp Med Biol 2007; 618: 229-44.

29. Helmark IC, Mikkelsen UR, Børglum J, Rothe A, Petersen MHC, Andersen O, et al. Exercise increases interleukin-10 levels both intraarticularly and peri-synovially in patients with knee osteoarthritis: a randomized controlled trial. Arthritis Res Ther 2010; 12: R126.

30. Lopes de Jesus CC, dos Santos FC, de Jesus LMOB, Monteiro I, Sant'Ana MSSC, Trevisani VFM. Comparison between intra-articular ozone and placebo in the treatment of knee osteoarthritis: A randomized, doubleblinded, placebo-controlled study. PLoS One 2017; 12: e0179185.

31. Bozbaș CT, Yilmaz M, Paşaoğlu E, Gürer G, İvgin R, Demirci B. Effect of ozone in Freund's complete adjuvant-induced arthritis. Arch Rheumatol 2018; 33: 137-42.

32. Mawsouf M, Maha El-Sawalhi M, Martínez-Sánchez G, Hebatalla Darwish A, Amira Shaheen A. Effect of ozone therapy on redox status in experimentally induced arthritis. Ozone Therapy Global Journal 2011; 1: $32-43$.

33. Motohashi H, Yamamoto M. Nrf2-Keap1 defines a physiologically important stress response mechanism. Trends Mol Med 2004; 10: 549-57. 